\title{
analyse de la fiabilité des barrages en terre
}

\author{
reliability analysis for earth dams
}

\author{
J.-L. FAVRE \\ Professeur, Laboratoire de Mécanique des Sols, Structures et Matériaux, CNRS URA $850^{\circ}$ \\ A. BEKKOUCHE \\ Maître Assistant, Institut National d'Enseignement Supérieur de Génie Civil, Tlemcen**
}

Rev. Franç. Géotech. n 53, pp. 45-54 (octobre 1990)

\section{Résumé}

L'article examine le développement des recherches sur la prise en compte des incertitudes dans l'analyse de la fiabilité des barrages en terre.

Après avoir souligné l'importance des ruptures, les auteurs montrent le poids particulier des incertitudes sur les terres pour les deux principaux états critiques (érosion et stabilité) et donnent une illustration détaillée pour l'érosion interne.

Ensuite, plusieurs techniques sont mises en œuvre pour mesurer, modéliser et contrôler les aléas sur les terres: I'analyse factorielle pour contrôler les zones d'emprunt, l'analyse de variance-covariance pour réduire les aléas sur les paramètres de projet et les modéliser, la géostatistique pour contrôler le compactage des terres et tenir compte de la position géographique des aléas. Toutes ces techniques contribuent à mieux connaître et prendre en compte la succession des incertitudes et des erreurs.

Enfin, les auteurs indiquent l'absence, malheureusement encore, de méthodes opérationnelles pour calculer le rôle de ces incertitudes et erreurs sur le résultat final. C'est dans ce sens que les efforts doivent aller.

\section{Abstract}

This paper examines the current research developments on uncertainty factors which play a crucial role in the analysis of earth dams reliability.

In emphasizing the importance of failure, the authors underline the particular role played by uncertainty on earth properties for 2 principal critical states (erosion and stability). A detailed illustration of internal erosion is also presented. In order to measure, modelise and control the hazards of earth, the following techniques are used : factorial analysis in order to control the borrow areas and the significance of the test samples, variance-covariance analysis in order to reduce hazards and modelize design parameters, geostatistics in order to control earth compaction and to take into account the geographic position of hazards. All of these techniques will contribute to a better understanding and manipulation of successive uncertainties and errors.

Finally, the authors suggest some possibilities of where future research should be oriented in order to develop operational methods for calculation the role of uncertainties and errors.

\footnotetext{
- Ecole Centrale de Paris, Grande voie des vignes, 92295 Chatenay-Malabry Cedex.
}

$\cdots$ RN 22, BP 230, Tlemcen, Algérie. 


\section{INTRODUCTION}

La fiabilité des barrages en terre tient à la grande expérience acquise par les projeteurs qui ont à tenir compte de l'ensemble des aléas liés au site et à l'ouvrage lui-même. Ces facteurs déterminent les mécanismes de ruine que le projeteur voudra rendre les plus improbables possibles. On peut apprécier l'importance relative de ceux-ci grâce à l'exploitation des statistiques sur les accidents et incidents de barrages. On estime qu'il existe près de 40000 grands barrages (en 1982: 16200 , en dehors de la Chine). Le nombre des ruptures reste important puisqu'il correspond à 1,5 rupture par an et le pourcentage des incidents est 2 à 7 fois plus grand, suivant le type d'ouvrage. Le nombre des ruptures, différent aussi d'un ouvrage à l'autre décroît fortement avec lâge de celui-ci et avec sa hauteur comme le montre des statistiques de SERAFIM. Enfin, les causes de rupture varient suivant l'âge de l'ouvrage cornme l'indique cette étude due à LEBRETON (tableau 1) sur les barrages en remblai qui représentent $70 \%$ du total des ouvrages.

$\mathrm{Si}$ de telles analyses ont été largement approfondies pour comprendre la pathologie des barrages, on concoit qu'elles ne puissent donner que des ordres de grandeur de la fiabilité d'un ouvrage, d'autant plus que chacun et son site sont un cas particulier. On devra utiliser une démarche déductive.

\section{MÉTHODE GÉNÉRALE D'ANALYSE}

\subsection{Les arbres de causes-conséquences}

L'étude de la pathologie des barrages en remblai permet de distinguer trois grands types de ruine ou états critiques :

- la submersion du barrage, pendant ou après la construction ;

- l'instabilité des talus amont et aval ;

- l'érosion interne du corps du barrage.

Pour chaque état critique, on analyse les actions ou les événements qui peuvent y conduire, ainsi que les diverses conséquences, avec leurs scénarios, qui peuvent en découler. On construit ainsi des arbres de causes-conséquences (fig. 1) dont on probabilise les diverses branches.

La somme des coûts des conséquences possibles, pondérés par les probabilités d'occurence de celles-ci, représente le risque total que l'on cherche à minimiser, pour un coût initial et un bénéfice donnés. Cette minimisation se fait par rapport à des paramètres de projet appelés alors paramètres de décision.

La submersion des barrages est avant tout un problème d'hydrologues et d'hydrauliciens et nous ne l'évoquerons pas ici bien que ses conséquences fassent intervenir les matériaux de remblai lors de l'érosion de surface des talus. Par contre les deux autres états critiques font intervenir la qualité des matériaux au niveau de l'arbre des causes.

Parmi celles-ci, il y a lieu de distinguer les causes premières qui, en première approximation, sont invariantes au cours du temps, et les événements initiateurs liés au temps et qui déclenchent les états critiques: - pour l'instabilité des talus, les causes premières sont les pentes (conception du barrage) et les propriétés mécaniques des matériaux de remblai, liées à leur nature et leur mise en ceuvre. Les événements initiateurs sont les sollicitations extérieures (séismes, chocs, etc.), les changements ou évolutions de régime hydraulique, l'évolution des propriétés mécaniques ou les déformations induisant de nouveaux champs de contraintes, etc. ;

- pour l'érosion interne, qui est un phénomène essentiellement évolutif, les causes premières sont les propriétés mécaniques et hydrauliques des matériaux compactés (érodabilité, perméabilité), et la conception et l'état des filtres et des étanchéités. Les événements initiateurs peuvent être les mouvements différentiels du corps du barrage, la modification du régime hydraulique, les actions extérieures ou P'évolution du matériau lui-même.

La figure 2 montre une telle analyse, qui pose en pratique des problèmes importants :

- détaillée, cette analyse apparaît comme un processus très complexe s'apparentant à la constitution de la base de connaissances d'un système-expert général pour les projets de barrages, mais trop simplifiée, elle perd beaucoup de son utilité ;

Tableau 1. - Ruptures de barrages de plus de $15 \mathrm{~m}$ pendant leur construction et leurs premières années (1964-1983) (d'après LEBRETON, 1985).

Table 1. - Failures of dams higher than $15 \mathrm{~m}$ during construction and after few years (1964-1983) (from LEBRETON, 1985).

\begin{tabular}{|c|c|c|c|c|c|c|c|}
\hline \multirow[t]{2}{*}{ Etat ou âge } & \multicolumn{3}{|c|}{ Nombre de ruptures } & \multicolumn{4}{|c|}{ Fréquence des ruptures } \\
\hline & Digues & Autres & $\begin{array}{l}\text { Ruptures } \\
\text { de vannes }\end{array}$ & Total & Digues & $\begin{array}{c}\text { Autre } \\
\text { en } 1 / 1000\end{array}$ & Total \\
\hline $\begin{array}{l}\text { En construction } \\
\text { Au } 1 \text { er remplissage et } \\
\text { pendant les } 5 \text { premiè- } \\
\text { res années } \\
\text { A } 1 \text { er remplissage et } \\
\text { pendant les } 10 \text { premiè- } \\
\text { res années }\end{array}$ & $\begin{array}{l}11^{(1)} \\
7^{(2)} \\
9^{(3)}\end{array}$ & $1^{(4)}$ & 1 & 12 & 1,2 & 0,8 & 1,7 \\
\hline
\end{tabular}

(1) 9 ruptures par submersion lors de crues et brêche, une par renard.

(2) 6 ruptures par renard et une par submersion de crue.

(3) de 6 à 10 ans: 1 rupture par submersion de crue et une par glissement de talus de la digue.

(4) Renversement d'un barrage-poids. 


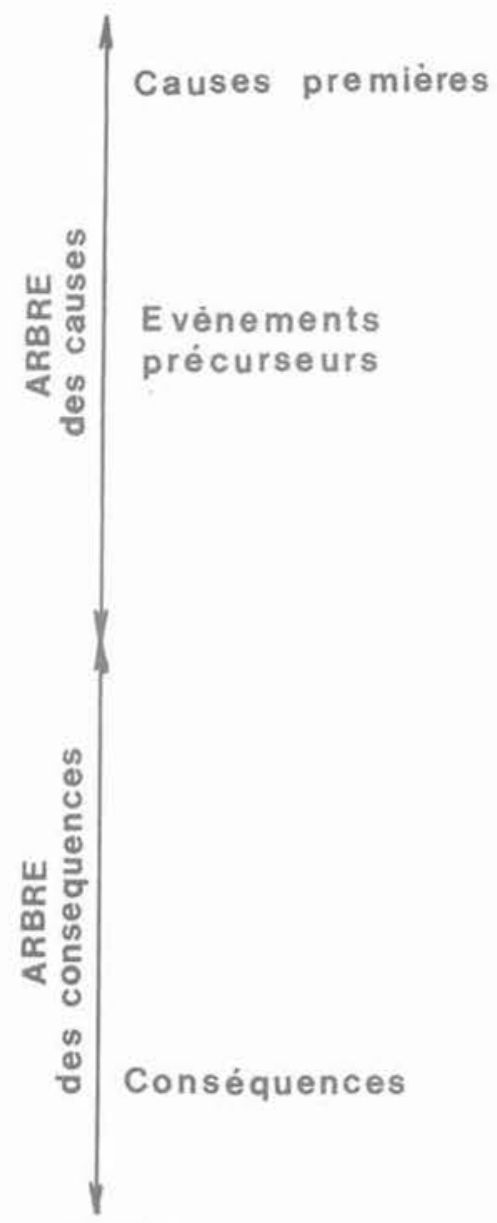

COUTS

RISQUE $\quad \sum_{1}^{4} P_{i} \cdot C_{i}$

COUT TOTAL $\mathrm{C}_{0}+\sum \mathrm{P}_{\mathbf{i}} \cdot \mathrm{C}_{\mathbf{i}}=\mathrm{C}_{\mathrm{T}}$

DECISION

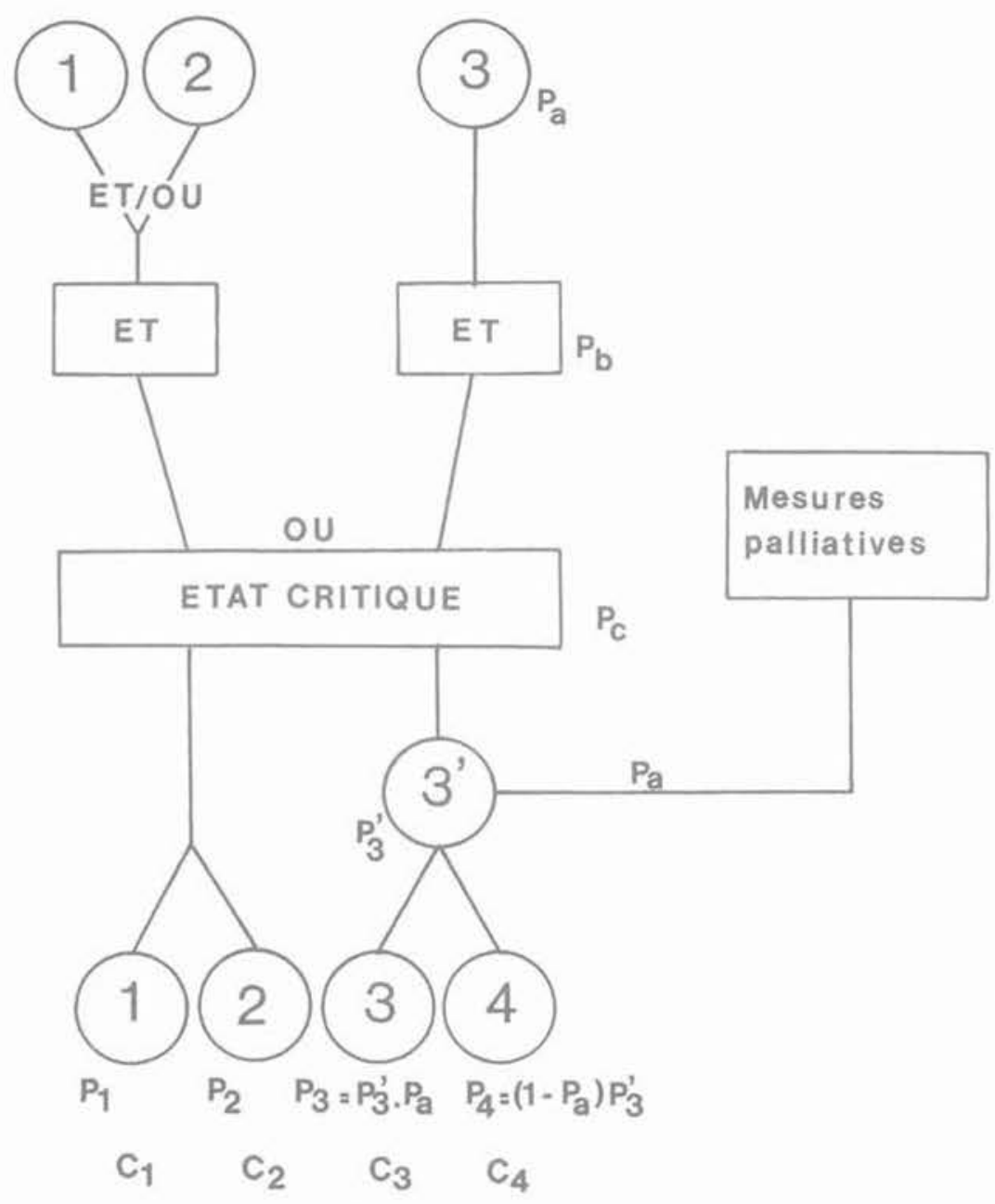

$$
a^{*} \frac{\partial c_{T}}{\partial a}=0=\frac{\partial c_{0}}{\partial a}+\frac{\partial}{\partial a} \sum P_{i} \cdot c_{i}
$$

Fig. 1. - Arbre de causes-conséquences.

Fig. 1. - Fault consequence tree.

- les probabilités de certains événements peuvent être difficiles, voire impossibles, à évaluer.

Le projeteur utilisera donc la méthodologie des arbres de causes-conséquences pour apprécier l'ensemble des événements qui peuvent provoquer un état critique. Il dégagera ceux dont les combinaisons sont les plus probables et calculera le poids de celles-ci sur l'état critique, grâce à la théorie de la fiabilité.

\subsection{Eléments de la théorie de la fiabilité}

Soit $M_{A}$, le moment agissant et $M_{R}$, le moment résistant maximum mobilisable. $M_{A}$ et $M_{R}$ sont des variables aléatoires, fonctions des différents événements, causes et incertitudes $X_{i}$ précédemment définis et de loi de densité de probabilité jointe $f_{x}\left(x_{i}, \ldots x_{n}\right)$. La probabilité de ruine peut s'écrire alors:

$$
\begin{aligned}
p_{r} & =\operatorname{Prob}\{g(X) \leq 0\} \\
& =\int_{D} f x\left(x_{1}, \ldots x_{n}\right) d x_{1} \ldots d x_{n} .
\end{aligned}
$$

où : $g(X)=M_{R}-M_{A}$ est la fonction d'état critique ou fonction de performance, $\mathrm{X}$ est le vecteur des variables $X_{i}$, dites de base, et $D=\left\{x_{i} \mid g\left(x_{i}\right) \leq 0\right\}$ le domaine de ruine dans l'espace des variables de base. 


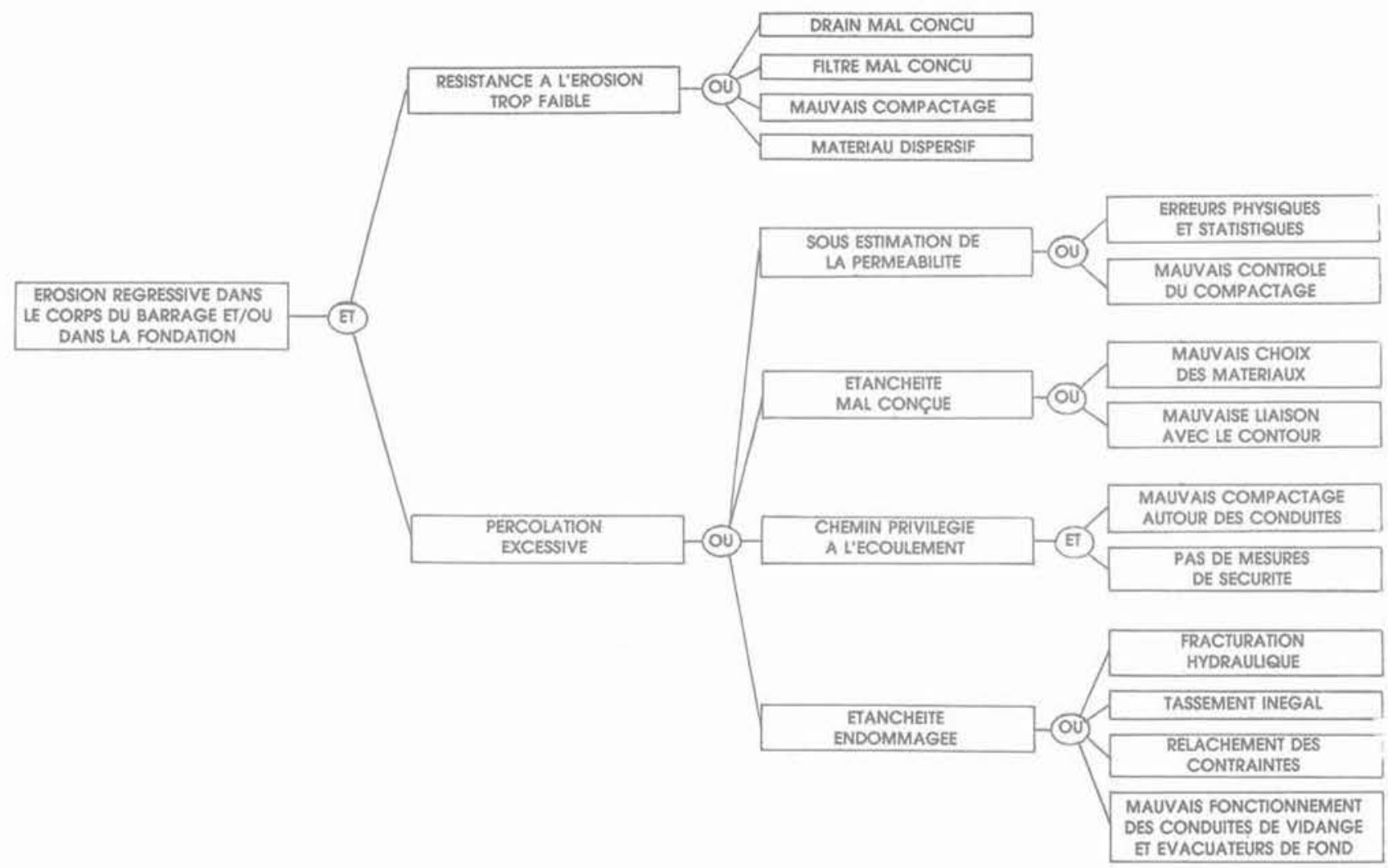

Fig. 2. - Arbre détaillé des causes pour l'érosion interne.

Fig. 2. - Detailled fault tree for internat erosion.

On définit l'indice de sécurité de Cornell par:

$$
\beta=E[g(X)] / \sigma_{g(X)},
$$

où : $E[g(X)]$ est l'espérance mathématique de $g(X)$ et et $\sigma_{\mathrm{g}(\mathrm{X})}$ son écart-type.

Sous certaines conditions (normalité et indépendance des $\mathrm{X}_{\mathrm{i}}$ et linéarité de la fonction de performance), la probabilité de ruine est exactement :

$$
P_{r}=\Phi(-\beta) \text {. }
$$

où : $\Phi$ est la fonction de répartition de la variable normale centrée réduite.

Ainsi, avec $m_{R}$ et $m_{A}$, les espérances mathématiques de $M_{R}$ et $M_{A}$, et $\sigma_{R}$ et $\sigma_{A}$, leurs écarts-types,

$$
\beta=\left(m_{R}-m_{A}\right) /\left(\sigma_{R}^{2}+\sigma_{A}^{2}\right)^{1 / 2} \text {. }
$$

En géotechnique, où les volumes concernés par les états critiques sont grands, le calcul des écarts-types des résistances et des sollicitations posent des problèmes particuliers : les variances des variables de base n'interviennent pas directement, mais diminuées par un facteur de réduction qui tient compte du fait que ces variables de bases sont, en vérité, des fonctions aléatoires de l'espace dont 2 valeurs proches sont corrélées. Il est donc de la première importance de connaître ces corrélations appelées fonctions d'autocorrélation, dont une des caractéristiques essentielles est la portée ou échelle de fluctuation, c'est-à-dire la distance à partir de laquelle 2 valeurs peuvent être considé. rées comme indépendantes.

Parmi ces variables, fonctions aléatoires de l'espace, il y a, au premier chef, les propriétés mécaniques et hydrauliques des matériaux de remblai. En effet, que ce soit parmi les causes premières ou parmi les événements initiateurs, celles-ci jouent un rôle essentiel. Ce sont donc en premier lieu les aléas sur ces propriétés qu'il faudra chercher à connaître (statistiques). réduire et contrôler. Et il faudra le faire en tenant compte de leur influence sur le comportement du barrage en construction puis en service, c'est-à-dire en modélisant l'effet des erreurs, des incertitudes et de la variabilité naturelle des matériaux sur le comportement de l'ouvrage (probabilités).

\section{RÉDUCTION DES ALÉAS SUR LES TERRES DE REMBLAI}

Dans un projet de barrage, chaque matériau utilisé a des propriétés bien définies que l'on choisit en tenant compte des matériaux effectivement disponibles dans les zones d'emprunt, et de leurs propriétés physiques et mécaniques à l'état naturel et après compactage. La reconnaissance des zones d'emprunt permet d'estimer les quantités de matériaux disponibles et leur homogénéité. Des contrôles de densité au fur 
et à mesure de l'édification du barrage permettent de vérifier la qualité du compactage. La statistique et la géostatistique offrent des méthodes d'analyse intéressantes, comme l'a montré BEKKOUCHE (1987) sur le cas du barrage de Grand'Maison.

\subsection{Contrôle des zones d'emprunt}

La reconnaissance de ces zones comporte des analyses granulométriques systématiques sur des forages verticaux implantés régulièrement sur tout le site. Puis, au vu de ces résultats, on choisit des échantillons représentatifs sur lesquels seront réalisés des essais de compactage, de perméabilité, de cisaillement et de déformabilité en laboratoire ou sur remblais d'essai. Les techniques d'analyse factorielle sont bien adaptée au traitement de ces résultats en grand nombre (FAVRE, 1980). Lorsqu'on veut condenser l'information contenue dans un tableau de données $\mathrm{X}$ ( $\mathrm{n}$ analyses granulométriques suivant $\mathrm{p}$ passants), on reconstitue ce tableau avec un nombre plus petit de données ( $n \times q, q=2$ à 3 ). On cherche dans lespace $\mathbb{R}^{p}$ des variables (ou $\mathbb{R}^{n}$ des individus) les axes successifs orthogonaux qui minimisent l'inertie du nuage des individus (ou des variables) : ils portent les vecteurs propres $\mathbf{u}_{\mathrm{a}}$ de $X^{\prime} X\left(\right.$ ou $v_{\mathrm{a}}$ de $X X^{\prime}$ ). On sait alors calculer les coordonnées $\mathrm{X}_{\mathrm{a}}$ des points du nuage dans ces axes, et on obtient la formule de reconstitution approchée du tableau X :

$$
\mathrm{X}^{\circ}=\sum_{1}^{\mathrm{q}} \sqrt{\mu_{\mathrm{a}}} \mathrm{v}_{\mathrm{a}} \mathrm{u}_{\mathrm{a}},
$$

où : $\mu_{\mathrm{a}}$ est la valeur propre associée au vecteur propre $u_{\mathrm{a}}$. Le taux d'information concentré sur l'axe factoriel :

$$
\mathrm{F}_{\mathrm{a}} \equiv \mathrm{u}_{\mathrm{a}} \text { est égal à } \mu_{\mathrm{a}} / \sum_{1}^{\mathrm{q}} \mu_{\mathrm{a}} \text {. }
$$

Ainsi, on peut connaître le pourcentage d'information que l'on perd quand on projette le nuage dans le plan des deux premiers axes factoriels pour juger de l'homogénéité des échantillons.

On peut aussi agréger les points du nuage en classes successives avec une règle de distance et représenter ces partitions successives par un arbre dont les niveaux des branches sont les distances entre classes ou niveaux de ressemblance.

Ainsi, pour le contrôle des terres à noyau du barrage de Grand'Maison, en utilisant comme variables les classes granulométriques de 20 à $63 \mathrm{~mm}$, de 5 à $20 \mathrm{~mm}$, de 0,1 à $5 \mathrm{~mm}$ et de moins de $0,1 \mathrm{~mm}$, sur 122 échantillons identifiés par 1 ou 2 lettres pour indiquer le puits ou la tranchée de prélèvement dans la zone d'emprunt et 2 chiffres pour indiquer sa profondeur en mètres, on obtient le résultat des figures 3 et 4 :

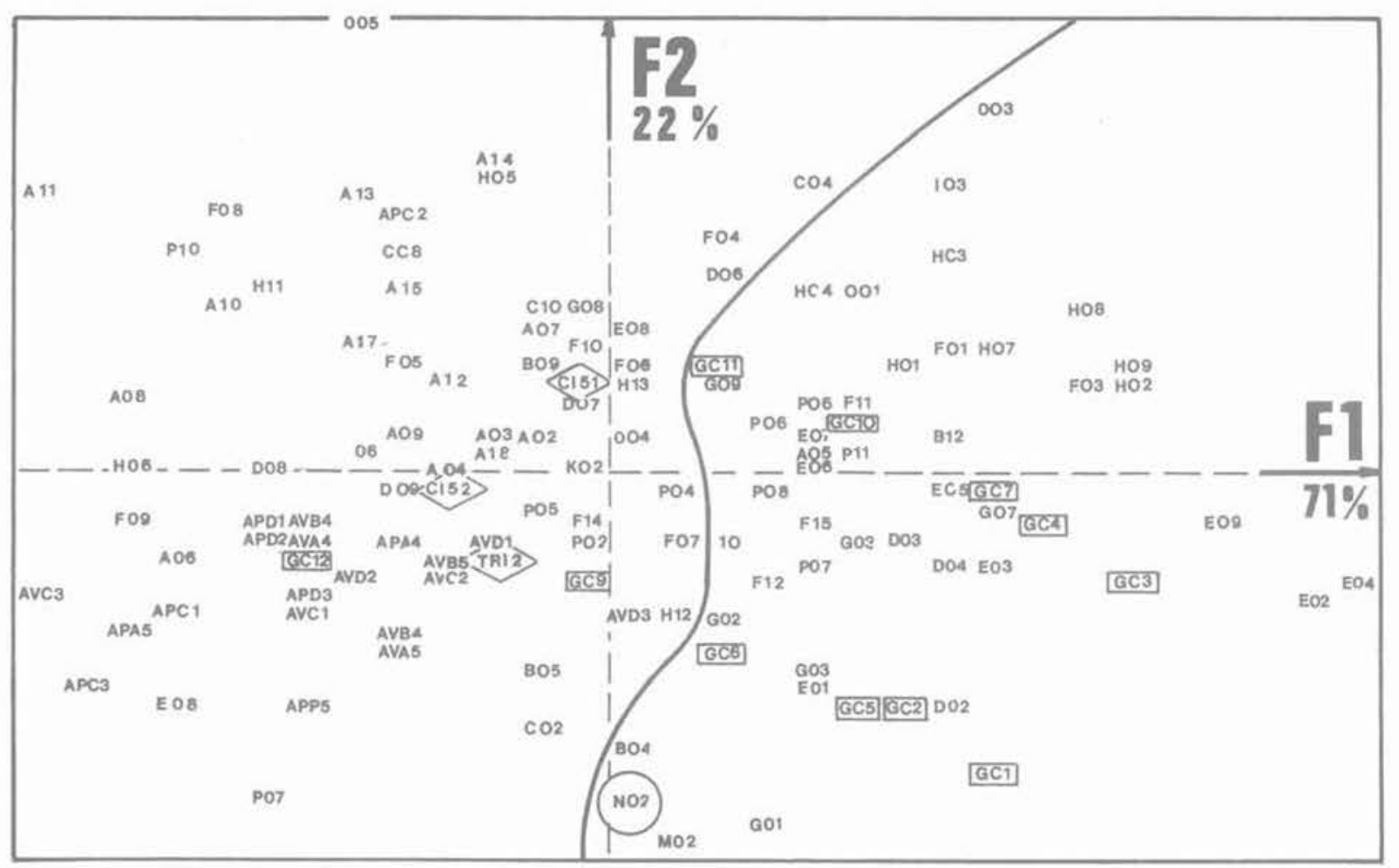

essais Proctor
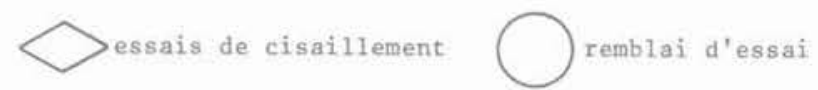

Fig. 3. - Terre à noyau de Grand'Maison. Analyse de la zone d'emprunt en composantes principales. Fig. 3. - Soil for the core of Grand'Maison dam. Principal component analysis of the borrow area. 


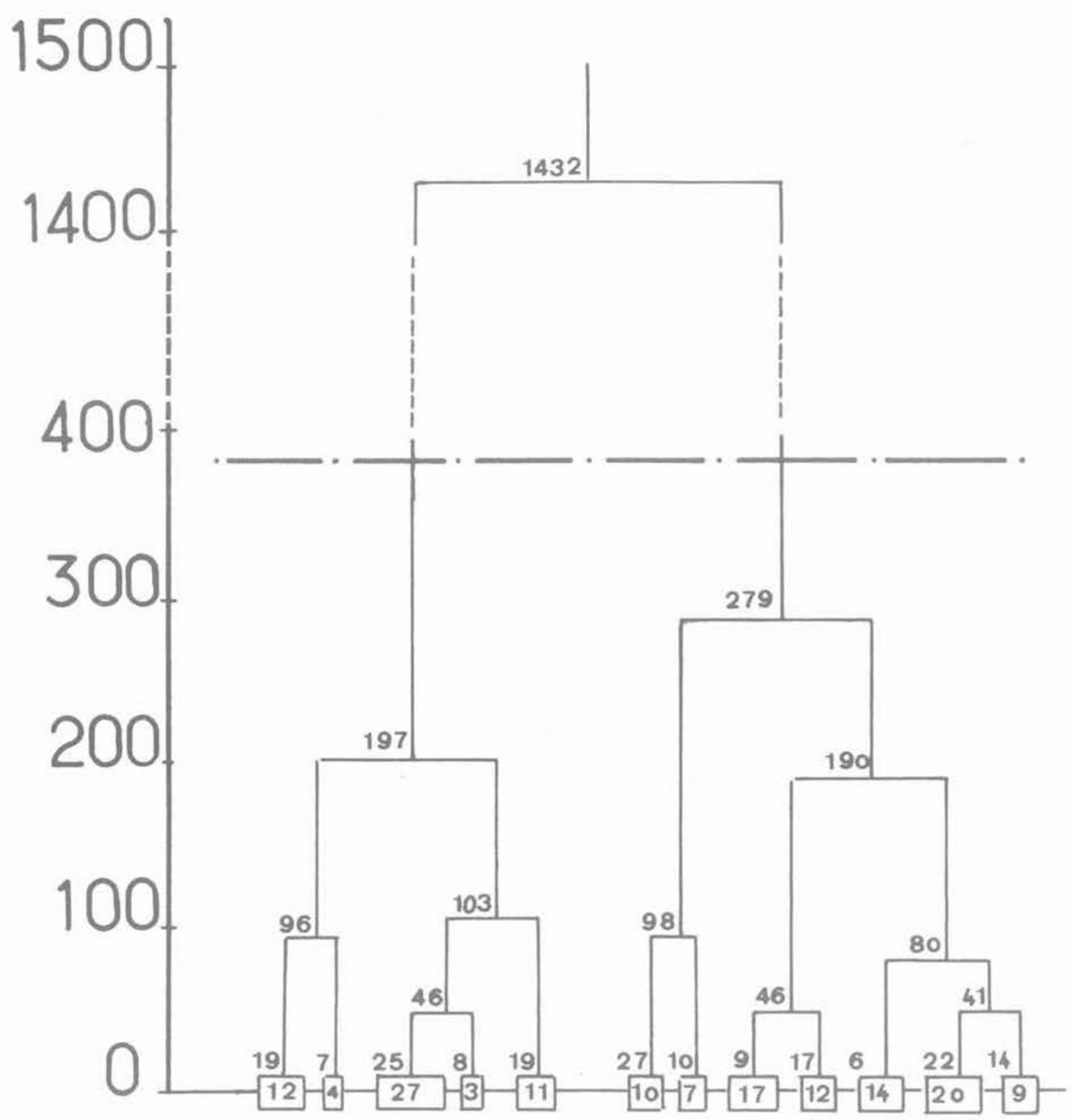

Fig. 4. - Terre à noyau de Grand'Maison. Analyse de la zone d'emprunt par classification hiérarchique ascendante. Fig. 4. - Soil for the core of Grand'Maison dam. Upward hierarchical classification of the borrow area.

- 2 classes de matériaux se différencient bien entre les niveaux 300 et 1500 (fig. 4), alors que l'EDF avait considéré une seule classe sur ce gite. Par contre, dans le premier plan factoriel (fig. 3) qui concentre $93 \%$ de l'information, cette partition ne semble se faire ni suivant la profondeur ni suivant la position géographique (code des puits) dans le gite. L'hypothèse d'une zone homogène se justifie (nuage compact) ;

- les échantillons retenus pour les essais en laboratoire apparaissent comme bien représentatifs du matériau car se situant au centre du nuage. Ceci est surtout vrai pour les essais de cisaillement (TRI et CI) et les essais Proctor (GC).
Cet exemple de contrôle de zones d'emprunt et du choix des échantillons montre qu'on pourrait conserver, pour la recherche des aléas suivants, les hypothèses suivantes: le matériau est homogène, les échantillons sont représentatifs ; il n'y aura pas de biais et de dispersion sur les variables du problème autres que ceux liés à la variabilité naturelle et à la mesure des propriétés des matériaux.

\subsection{Essais de laboratoire}

En général, on ne sait pas séparer ce qui tient de la variabilité de la valeur naturelle $\mathrm{X}_{\mathrm{N}}$ d'un paramètre, de ce qui tient des erreurs de mesure $e_{m}$. Ainsi, la valeur mesurée s'écrit :

$$
X_{m}=X_{N}+e_{m}
$$


C'est la valeur naturelle, "vraie ", qui intéresse le projeteur. Il la modélise par une variable aléaloire en écrivant :

$$
\begin{aligned}
& E\left[X_{N}\right]=E\left[X_{m}\right]-E\left[e_{m}\right] \\
& \operatorname{Var}\left[X_{N}\right]=\operatorname{Var}\left[X_{m}\right]-\operatorname{Var}\left[e_{m}\right]
\end{aligned}
$$

L'espérance et la variance des erreurs peuvent être avantageusement réduites lorsquion sait en séparer les causes (facteurs d'influence intervenant par 2 modalités ou plus). Ainsi, pour le barrage de Grand'Maison, l'angle de frottement interne $\phi$ des terres à noyau été estimé à partir de 10 mesures, soit à la boîte soit au triaxial (facteur A), en condition soit drainée soit non drainée (facteur B), sur un matériau écrété soit à $63 \mathrm{~mm}$ soit à $20 \mathrm{~mm}$ (facteur C) et pour des compacités initiales variables (facteur D) (fig. 5 et tableau 2).

L'analyse de variance-covariance permet de tester l'influence des différents facteurs, de faire appaître les biais que chacun introduit (espérance d'erreur) et de réduire la variance d'erreur. Ainsi, il a été trouvé que seul le facteur A (type d'essai) avait une influence significative. Le projeteur pourra alors passer du modèle "brutal " :

$$
\phi=40,2^{\circ}+\epsilon, \quad \sigma_{\epsilon}=3,99^{\circ},
$$

au modèle :

$$
\phi=37,5^{\circ}+\epsilon, \quad \sigma_{\epsilon}=3,09^{\circ},
$$

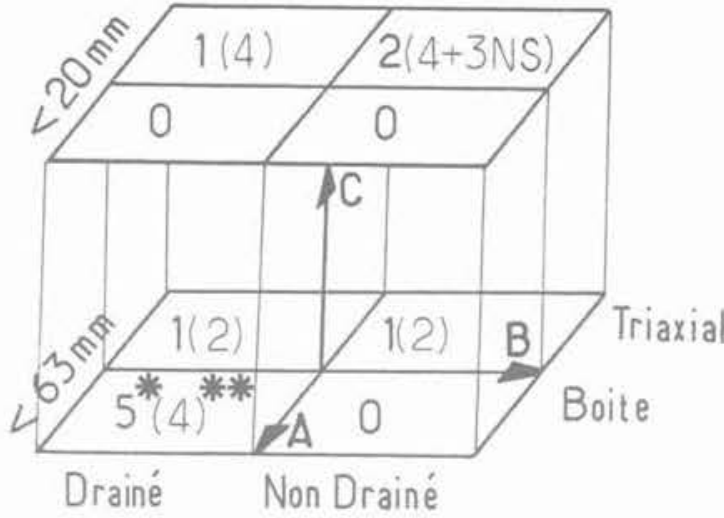

*nombre de mesures de $\varnothing_{\text {pir }}$

** nombre de mesures de $\tau_{\text {r }}^{\text {r }}$

Fig. 5. - Répartition des essais suivant les modalités des différents facteurs d'influence.

Fig. 5. - Test distribution according to the modes of the various influence factors.

si l'on considère que seule la boîte, à l'encontre du triaxial, introduit un biais de représentativité.

Les caractéristiques du modèle $\left(\bar{\phi}=37,5^{\circ}\right.$, $\sigma_{\phi}=3,09^{\circ}$ ) ne sont qu'estimées à partir de 10 valeurs. Il faudra leur adjoindre des intervalles ou des seuils de confiance en utilisant la théorie de l'estimation. Ici, sous certaines hypothèses, on peut assurer que :

$$
\begin{aligned}
& \text { Prob }\left[\phi \leq 35,7^{\circ}\right\}=10 \% \\
& \text { Prob }\left\{\sigma_{\phi} \geq 4,54^{\circ}\right\}=10 \%
\end{aligned}
$$

\begin{tabular}{|c|c|c|c|c|c|c|c|c|c|c|}
\hline \multirow[b]{3}{*}{$\mathrm{N}$} & \multicolumn{6}{|c|}{ Facteurs d'influence } & \multicolumn{4}{|c|}{ Paramètres continus } \\
\hline & \multicolumn{2}{|c|}{ A } & \multicolumn{2}{|c|}{ B } & \multicolumn{2}{|c|}{ C } & \multirow{2}{*}{$\frac{W}{\%}$} & \multirow{2}{*}{$\frac{\gamma_{d i}}{K N / M^{3}}$} & \multirow{2}{*}{$\frac{\phi}{\text { degré }}$} & \multirow{2}{*}{$\frac{\mathrm{C}}{\mathrm{KPa}}$} \\
\hline & TRI & $B C$ & $C D$ & CND & $63 \mathrm{~mm}$ & $20 \mathrm{~mm}$ & & & & \\
\hline 1 & & $x$ & $\mathrm{x}$ & & $x$ & & 13,20 & 19,6 & 48 & 0 \\
\hline 2 & & $x$ & $x$ & & $x$ & & 8,17 & 20,1 & 46 & 0 \\
\hline 3 & & $x$ & $x$ & & X & & 9,2 & 18,9 & 46 & 0 \\
\hline 4 & & $\mathrm{x}$ & $x$ & & $x$ & & 10,07 & 18,8 & 40 & 0 \\
\hline 5 & & $x$ & $\mathrm{x}$ & & $x$ & & 10,29 & 19,4 & 37,5 & 14 \\
\hline 6 & $x$ & & $x$ & & $x$ & & 10,6 & 18,9 & 38 & 0 \\
\hline 7 & $\mathrm{X}$ & & & $x$ & $x$ & & 10,6 & 20,25 & 37,5 & 1,5 \\
\hline 8 & $x$ & & $\mathrm{X}$ & & & $x$ & 11,73 & 19,85 & 40 & 0 \\
\hline 9 & $x$ & & & $x$ & & $x$ & 11,18 & 19,85 & 37 & 5 \\
\hline 10 & $x$ & & & $x$ & & $x$ & 11,81 & 20 & 35 & 8 \\
\hline
\end{tabular}

Tableau 2. - Essais de cisaillement sur les terres à noyau.

Table 2. - Shear tests on core soil. 
On devra tenir compte de tels seuils dans les calculs de probabilité d'états critiques.

\subsection{Contrôle de réception des terres}

La définition des critères de réception est habituellement faite sans référence à la position des points de contrôle dans le remblai. C'est pourtant cette prise en compte qui permet de caractériser la variabilité naturelle des propriétés des terres compactées grâce aux fonctions d'autocorrélation.

Soit $t$ le point de coordonnées $(x, y, z), t+\tau$ le point de coordonnées $(x+\Delta x, y+\Delta y, z+\Delta z)$ et $X_{N}$ la valeur naturelle considérée comme fonction aléatoire de l'espace. On peut souvent considérer que la fonction d'autocorrélation ne dépend que de la distance $\tau$ entre points et pas de leur position absolue t. Elle s'écrit alors :

$$
\mathrm{BX}_{\mathrm{N}}(\tau)=\operatorname{Cov}\left[\mathrm{X}_{\mathrm{N}}(\mathrm{t}), \mathrm{X}_{\mathrm{N}}(\mathrm{t}+\tau)\right],
$$

ou encore :

$\rho(\tau)=\mathrm{BX}_{\mathrm{N}} / \operatorname{Var}\left[\mathrm{X}_{\mathrm{N}}\right]$, le coefficent de corrélation.

On peut, sur la base des mesures, si elles sont faites suivant un maillage suffisamment serré, du moins localement, adopter des modèles simples comme le modèle triangulaire (a) :

$$
\begin{aligned}
\rho=1-\tau / \theta, & \tau<\theta \\
\rho=0 \quad, & \tau>\theta,
\end{aligned}
$$

ou le modèle exponentiel (b) :

$$
\rho=\exp (-2 \tau / \theta)
$$

où : $\theta$ est la portée ou l'échelle de fluctuation (fig. 6).

On a ainsi analysé les paramètres granulométriques (1 000 échantillons) et de compactage (250 échantillons) du noyau du barrage de Grand'Maison (fig. 7 et 8), paramètres pouvant entrer indirectement dans un modèle d'érosion interne (calcul de la perméabilité puis des gradients hydrauliques) ou d'instabilité (calcul des caractéristiques de cisaillement) :

- les figures 7a et 8 a montrent les histogrammes permettant d'estimer l'espérance et la variance des paramètres ;

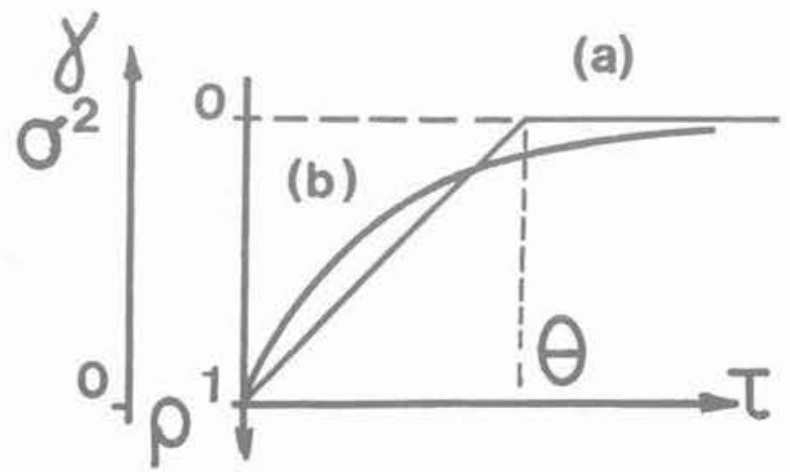

Fig. 6. - Fonctions d'autocorrélation. Fig. 6. - Autocorrelation functions.

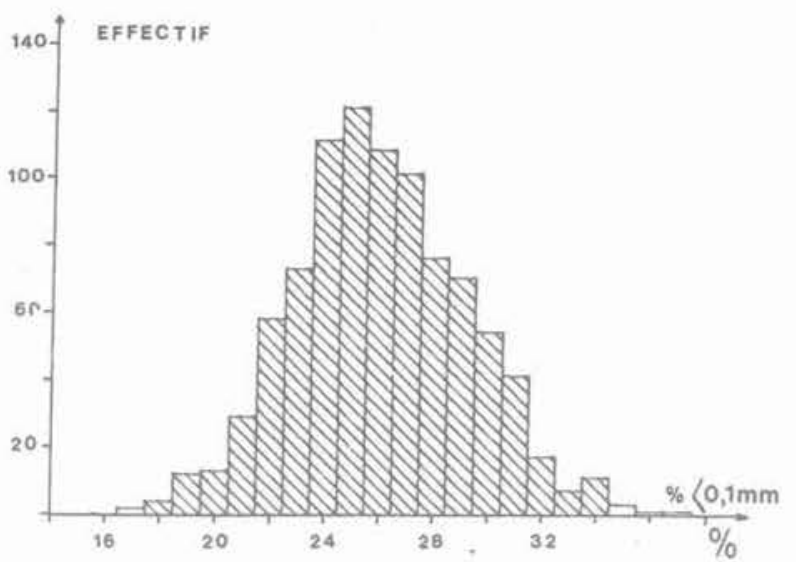

Fig. 7a. - Histogramme du $\%<0,1 \mathrm{~mm}$. Fig. $7 a$. - Histogram of $\%<0,1 \mathrm{~mm}$.
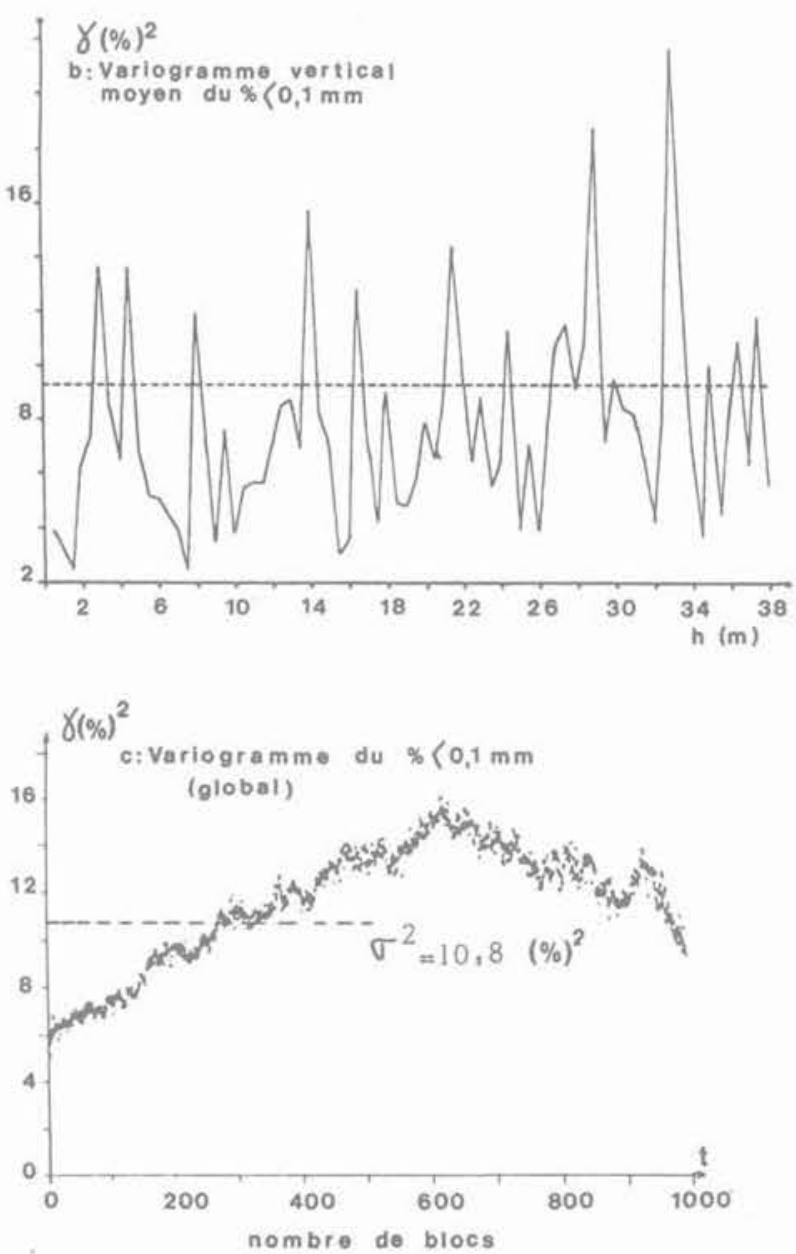

Fig. $7 b$ et c. - Variogrammes du $\%<0,1 \mathrm{~mm}$. Fig. $7 b$ et $c$. - Variograms of $\%<0,7 \mathrm{~mm}$.

- les figures $7 \mathrm{~b}$ et $8 \mathrm{~b}$ montrent leurs variogrammes (coefficient d'autocorrélation à une transformation linéaire près - voir figure 6 - en fonction de l'espace. Leur forme est très erratique ; il est impossible d'en tirer un modèle ou même de faire une interprétation ; 


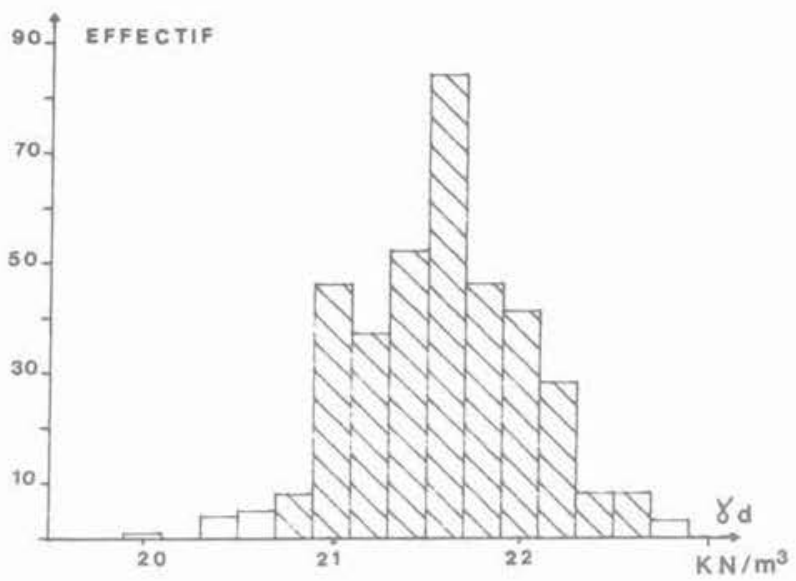

Fig. 8a. - Histogramme du poids volumique sec. Fig. $8 a$. - Histogram of the dry volumetric weight.

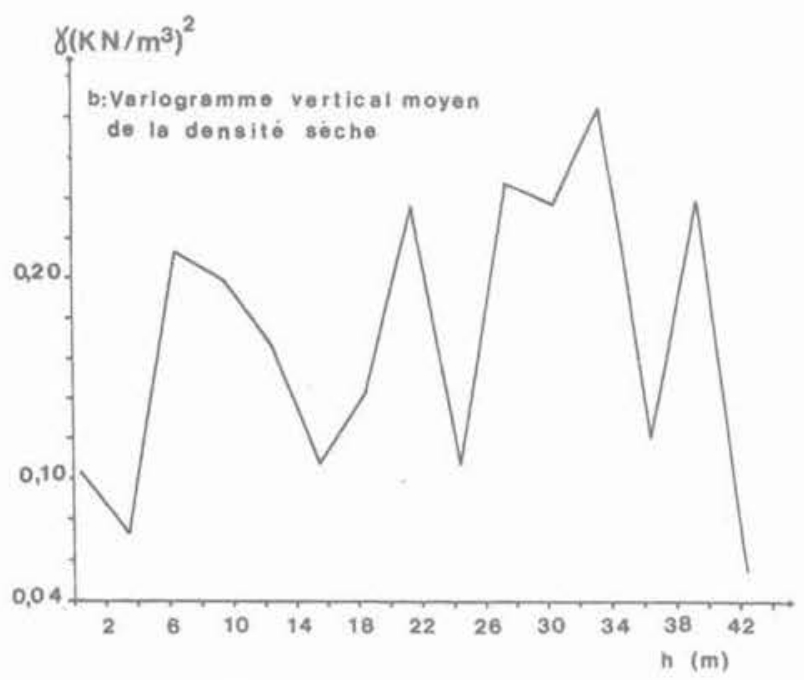

$\gamma\left(\mathrm{KN} / \mathrm{m}^{3}\right)^{2}$

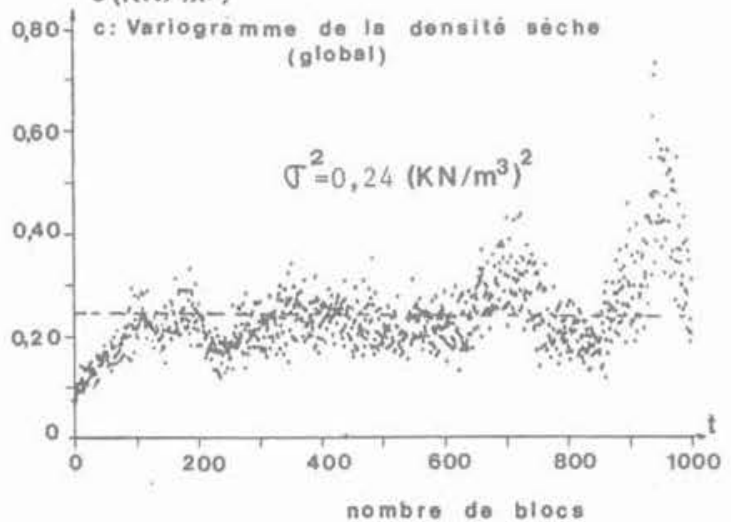

Fig. 8b. et c. - Variogrammes du poids volumique sec. Fig. $8 b$. et c. - Variograms of the dry volumetric weight.

- les figures $7 c$ et $8 c$ montrent les variogrammes en fonction du temps, c'est-à-dire de la chronologie de mise en place des terres. Ils présentent des formes régulières et usuelles (SOULIÉ, 1983), comme la présence systématique d'un fort effort de pépite (ordonnée à l'origine) relative en grande partie à l'erreur commise en assimilant la valeur unique mesurée à la valeur moyenne du "bloc " des terres mises en place. Le variogramme du $\%<0,1 \mathrm{~mm}$ présente un effet de trou significatif d'une ségrégation inhérente à l'emploi d'un matériau à granulométrie très étalée. Cette ségrégation a été localisée dans les 20 derniers mètres de la dernière campagne de mise en place;

- l'étude a montré que les points de contrôle étaient sinon en nombre insuffisant tout au moins à implantation géographique mal adaptée pour une véritable recherche des échelles de fluctuation.

\section{INFLUENCE}

\section{DE LA VARIABILITÉ DES MATÉRIAUX SUR LE COMPORTEMENT DU BARRAGE}

Pour pousser à son terme l'analyse des probabilités des états critiques d'un barrage, il faut pouvoir calculer l'influence de la variabilité naturelle des propriétés des matériaux de remblai, des incertitudes associées et des erreurs de mesure. Cette analyse est d'ailleurs également indispensable pour les analyses décisionnelles relatives aux reconnaissances et aux contrôles, puisque l'on peut tolérer plus d'incertitudes quand le risque est faible,

Les modèles de calcul des états critiques ne sont pas, à l'heure actuelle, encore bien établis. Les études réalisées jusqu'à présent montrent qu'il est indispensable de tenir compte de la variabilité naturelle spatiale des propriétés des matériaux. On peut l'admettre aisément si l'on pense que chaque état critique met en jeu un certain volume de matériau et que les propriétés moyennes sur ce volume ont une dispersion moindre que les propriétés du matériau en chaque point du massif (c'est ce qu'on appelle une moyenne locale et un effet de réduction de variance d'autant plus grand que le volume est grand devant l'échelle de fluctuation). Le souci d'en tenir compte dans les calculs probabilistes est encore récent et peu de travaux lui ont encore été consacrés. Pour certains aspects, la méthodologie de l'approche probabiliste a été dégrossie : travaux de VANMARCKE (1977), de GENEVOIS (1984), et MADHAVI (1985) pour les calculs de stabilité ; travaux de BOULEFKHAD (1986) pour les calculs de déformations. Pour d'autres (écoulements), les travaux sont en cours, et leurs chances de succès sont bonnes. Pour d'autres enfin (érosion interne), on ne dispose pas de modèle d'analyse déterministe utilisable pour guider l'analyse probabiliste et le succès est moins assuré.

L'absence de méthodes de calcul opérationnelles pour l'analyse probabiliste du comportement des corps de barrages en terre constitue actuellement l'un des principaux obstacles au développement de méthodes d'optimisation des projets de barrages sur la base de la théorie de la décision. C'est pour permettre de tels calculs, qui peuvent s'appliquer également à d'autres types d'ouvrages, que des travaux ont été engagés, au cours des dernières années, tant à l'Ecole Centrale de Paris qu'au Laboratoire Central des Ponts et Chaussées. 


\section{CONCLUSION}

Les méthodes d'analyse des données peuvent dès à présent être utilisées pour lanalyse des sites d'emprunts et le contrôle du compactage et de l'homogénéité des corps de barrages en terre. Par contre, les analyses de risques nécessitent des outils probabilistes dont le développement est seulement en train de se faire.

On peut penser que lanalyse des risques sera un jour un outil opérationnel dans les mains des projeteurs et gestionnaires de projets. Mais l'expérience des ingénieurs, l'auscultation de l'ouvrage en construction puis en service et le jugement des experts resteront toujours essentiels pour garantir la fiabilité des ouvrages. L'analyse statistique et probabiliste n'est qu'un outil, qui ne vaut que par la qualité des données analysées et des modèles utilisés. De grands progrès restent à faire, mais ils ne peuvent remplacer la formation et le perfectionnement des spécialistes des barrages en terre.

\section{REMERCIEMENTS}

Les auteurs remercient l'EDF qui leur a permis de travailler sur les données de Grand'Maison et plus spécialement M. DOUILLET qui a suivi ce travail dans le cadre du Gréco Géomatériaux, ainsi que M. MAGNAN du LCPC qui s'y est aussi intéressé.

\section{BIBLIOGRAPHIE}

BEKKOUCHE A. (1987), Sécurité des grands barrages en terre. Approche probabiliste des problèmes d'écoulement liés aux reconnaissances et contrô. les. Thèse de Doctorat, Ecole Centrale Paris, 380 p.

BOULEFKHAD T. (1986), Influence de la variabilité des sols sur le calcul des tassements des fonda. tions superficielles. Thèse de Docteur-Ingénieur, Univ. de Clermont II, 153 p. + annexes.

DORE M. (1986), Introduction et enseignements tirés des accidents. Electricité de France, Journées d'étude sur les barrages en argile. Chambéry, 14-15 mai $1986,19 \mathrm{p}$.

FAVRE J.L. (1980), Milieu continu et milieu discontinu. Mesure statistique indirecte des paramètres rhéologiques et approches probabiliste de la sécu. rité. Thèse de Docteur ès Sciences, Univ. P. et M. Curie (Paris VI), $500 \mathrm{p}$. + volume de figures.

FAVRE J.L., MAGNAN J.P., DOUILLET G. (1988), Analyses statistiques et modèles probabilistes pour les barrages en terre. Etat actuel et perspectives. Colloque technique * Réflexions sur l'économie de réalisation des barrages: leçons d'expérience ". Comité Français des Grands Barrages. Paris, 19 avril 1988, $12 \mathrm{p}$.

GENEVOIS B. (1984), Approche probabiliste de la force portante d'une fondation. Thèse de DocteurIngénieur, Ecole Centrale Paris, 150 p.

LEBRETON A. (1985), Les ruptures et accidents graves de barrages de 1964 à 1983. La Houille Blanche, Paris, $n^{\circ} 6 / 7$, pp. 529-544.

MAGNAN J.P. (1982), Les méthodes statistiques et probabilistes en mécanique des sols. Presses de l'Ecole Nationale des Ponts et Chaussées, Paris, $203 p$

MADHAVI C. (1985), Analyse probabiliste du comportement des sols et des ouvrages : évaluation des risques dans les études géotechniques de remblais sur sols mous. Thèse de DocteurIngénieur, Ecole Nationale des Ponts et Chaussées, Paris, $180 \mathrm{p}$.

POST G., LONDE P. (1954), Les barrages en terre compactée. Pratiques américaines. GauthierVillars, Paris, $185 \mathrm{p} .+$ annexes.

SERAFIM J.L. (1981), Safety of dams judged from failures. Water Power and Dam Construction, Dec. 1981, pp. 32-35. Rectificatif : May 1982, p. 49 .

SOULIÉ M., FAVRE M., KONRAD J.M. (1983), Analuse géostatistique d'un novau de barrage tel que construit. Revue Canadienne de Géotechnique, vol. $20, n^{\circ} 3$, pp. 453-467.

VANMARCKE E.H. (1978), Reliability of earth slopes. ASCE, Journal of Geotechnical Engineering, vol. 103, n GT11, pp. 1247-1265. 